\title{
Optimization of the Fibrous Component Absorption Performance of Hygienic Product Absorbent Core
}

\author{
S. Sayeb ${ }^{*}$, M. Ben Hassen and F. Sakli \\ Textile Research Unit, ISET of Ksar Hellal, Tunisia
}

\begin{abstract}
Absorbency has been always regarded as one of the most important features desired by consumers in disposable diapers. Absorption and retention capacity of a wood mixture pulp (fluff) and other fibers (acrylic and viscose), designed for production of the core of disposable diapers were studied. The kinetics of absorption as other main performance parameter of these products was also studied. The measurement of the performance parameters was carried out in the broad range of component weight ratios with a $\mathrm{NaCl}$ solution. The incorporation of these fibers was carried out also in the presence of super absorbent polymer particles. The behaviour of the components was found to improve absorption and retention capacity and also to cause a rise of absorbency rate. The insertion of acrylic fiber seems to limit the occurrence of the gel blocking phenomenon.
\end{abstract}

Keywords: Absorbent core, kinetic of absorption, absorption capacity, retention, disposable diapers.

\section{INTRODUCTION}

Many varieties of hygienic products are manufactured. Many of them have as main component, an absorbent core that contains cellulosic fibers from wood pulp (fluff) in absorbent core to absorb rapidly and hold large quantities of fluid is an important requirement. The absorbent performance of the final product is defined by the performance of the components i.e. absorption capacity, liquid holding ability, and transport of liquid through the absorbent core [4-

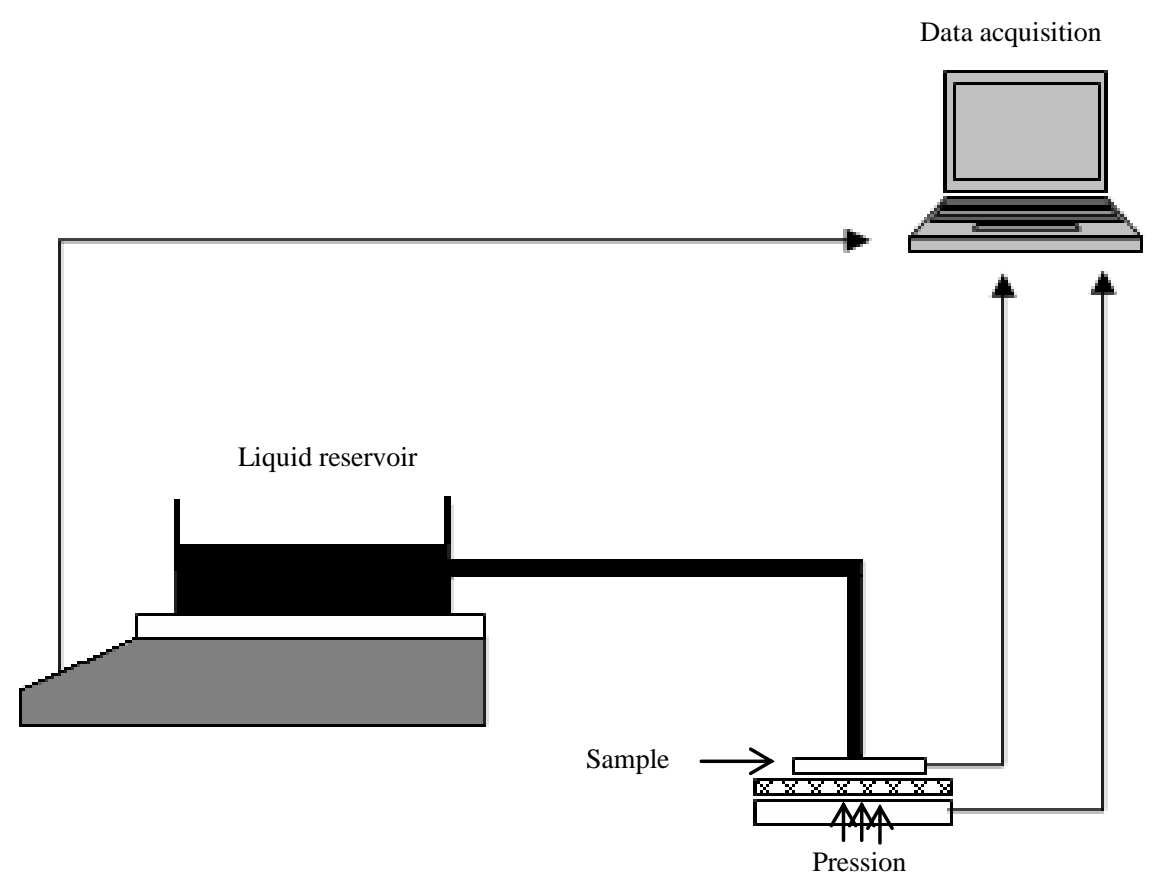

Fig. (1). Device used for absorption kinetics.

which super absorbent particles (synthetic polymer from the group polyacrylates) are spread [1-3]. The ability of the

*Address correspondence to this author at the Textile Research Unit, ISET of Ksar Hellal, 42 Avenue Farhat Hached kalaa kebira 4060, Tunisia; Tel: (+216) 97456 908; Fax: (+216) 73475 163;

E-mails: soumaessayeb@yahoo.fr, soumasayeb@hotmail.com
7]. Among these parameters, mechanical and physical properties of fiber, the resilience, deformation in the web without and under pressure, influence the absorptive behavior of the product, because the absorption properties involves not only swelling of hydrophilic fiber but also imbibitions of liquid in interstitial voids and capillaries and spreading of liquid in the core [8-11]. 
Nonwoven webs

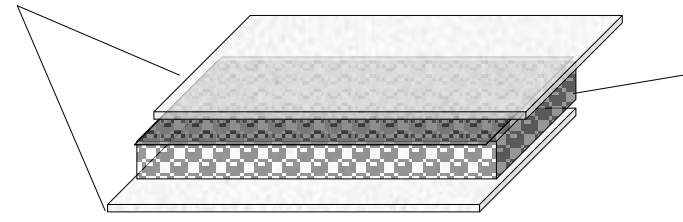

Absorbent core:

Mixture of fluff $+($ viscose/acrylic $)+$ SAP

Fig. (2). Model of laboratory samples.

The purpose of this study is to optimize absorbent core composition and particularly the fiber component by introducing other fibers (viscose and acrylic) at different ratios between them. The behavior of the absorbent core with super absorbent polymer (SAP) should enable gel blocking to occur. The performances tested are absorption capacity, retention and absorption kinetics which can inform us about the core ability to absorb, store and spread liquid.

\section{MATERIEL AND METHODS}

\section{Material Characteristics}

The materials used for the experiment were bleached soft wood fibers from STORA fluff LKC (mean length $2.7 \mathrm{~mm}$ ), HySorb B7160 super absorbent polymer based on sodium acrylate (BASF), commercial fibers of viscose (1.7dtex) and acrylic (1.3 dtex).

\section{Experiments}

Total absorption capacity and retention were realized according to STN2: $117 / 87$ standards for total absorbency and retention measurement. The liquid test used is a $0.9 \%$ $\mathrm{NaCl}$ solution.

The absorption capacity measures the liquid quantity remained associated to the material after a time of impregnation and draining. The retention measures the liquid quantity after sample centrifugation. The sample was previously impregnated and drained.

The principle of absorption kinetics measurement is based on mass variation of the absorbed solution by the sample as function of time. In fact, the tested sample is put under a pressure of $0.7 \mathrm{psi}$, and then a fixed volume of $20 \mathrm{ml}$ is absorbed. The absorbed quantity evolution as a function of time is recorded (Fig. 1).

The experiment was carried out in two stages:

1. Influence of fibers (viscose/ acrylic) on the absorbency performance of their mixture with fluff.

2. Influence of the blends (fluff + viscose/acrylic) with super absorbent on core performance.

Different tests were carried out on laboratory samples; the absorbent structure model is shown in Fig. (2). Samples for the test were prepared by mixing all the components in the blender and placed between two nonwoven webs. Mixtures of fluff with viscose/acrylic were realized according to different ratios: 90/10, 80/20, 70/30, 60/40 and 50/50.

\section{RESULTS AND DISCUSSION}

\section{Influence of Viscose/Acrylic Fibers on Absorption Performance of their Mixture with the Fluff}

The results of the experiments are presented on Fig. (3). The data shows that the values of absorption capacity and retention for the mixture of fluff with acrylic and viscose are higher than the ones for the fluff alone. The improvement reaches the maximum for a ratio of $80 / 20$ for a blend with viscose and for a ratio of 90/10 for acrylic. The retention values are equal to the retention value of fluff alone. This points out that the importance of physical structure that equals the swelling properties of fluff fiber. It is known that the resilience of acrylic fibers does not contribute to

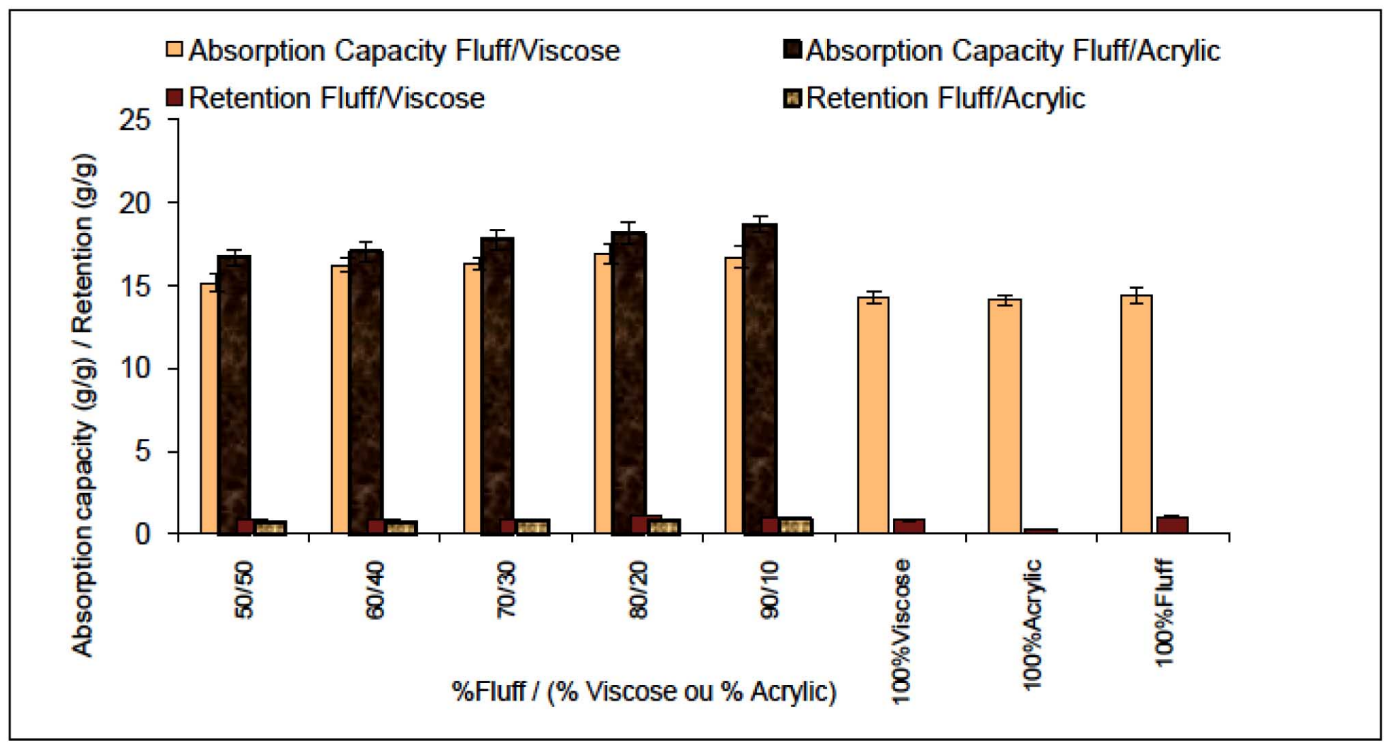

Fig. (3). Influence of viscose/acrylic fibers on absorption capacity and retention of mixture with the fluff. 


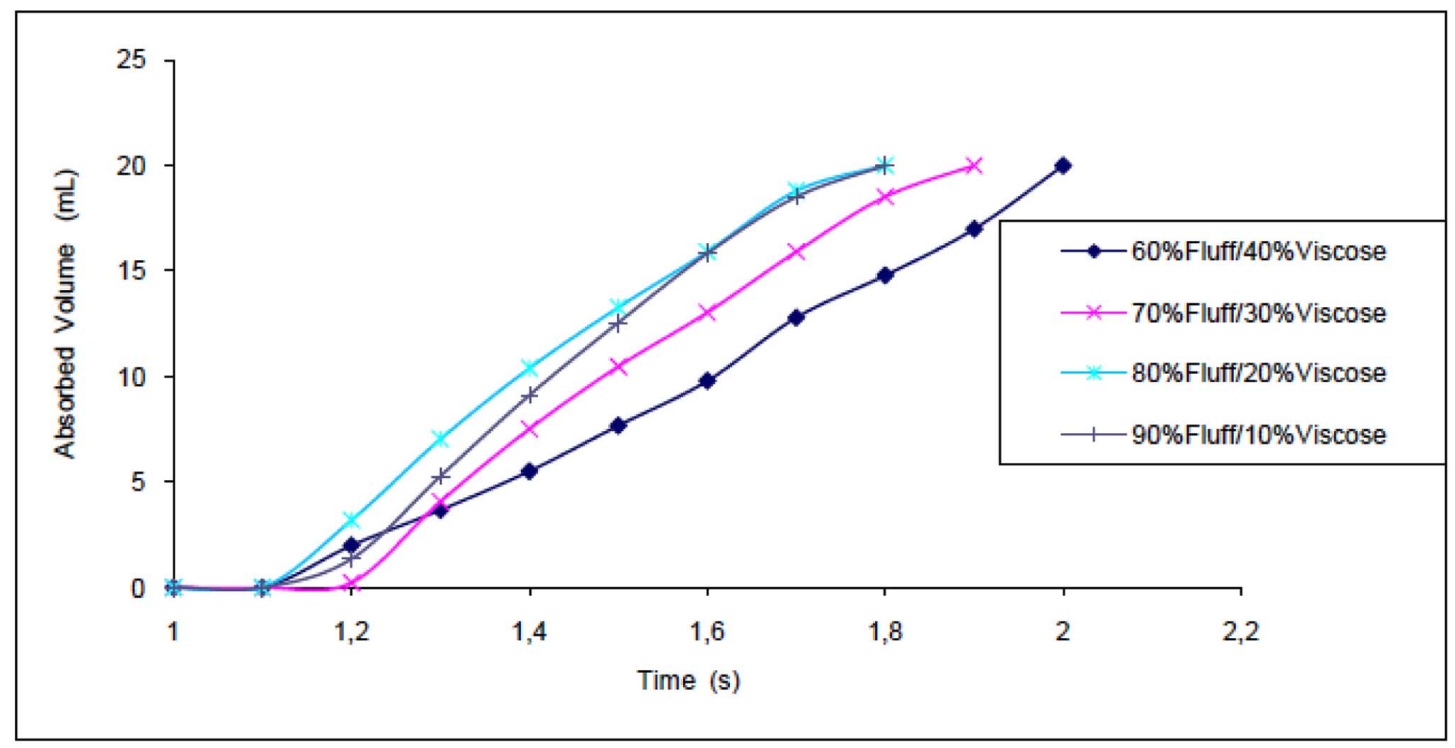

Fig. (4). Influence of viscose fiber on absorption kinetic of mixture with the fluff.

structure deformation in the wet state which enhances absorption and retention. In fact, it generates high volume which produces more porous structure and consequently higher value of absorption capacity $[6,10,12]$.

Moreover, absorption kinetics measurements confirm these statements. Figs. (4 and 5) show how the fibrous core absorbs the volume injected. Mixture with viscose records the best performance for the ratios $80 / 20$ and $90 / 10$ and this is similarly reached with acrylic blend.

By generating more porous structure, acrylic fibers improve liquid propagation and imbibitions through capillaries to transfer the liquid within absorbent core.

\section{Influence of Viscose/Acrylic Fibers on Absorption Performance of their Mixture with the Fluff and Superabsorbent}

During these experiments, third party fixed super absorbent ratio to $25 \%$. Fig. (6) shows that the ratio $80 / 20$ has the best absorption capacity values. Ratios of 70/30 and
90/10 record absorption capacity values equal to the ones obtained for fluff alone. But a ratio of 50/50 has lower performance than the fluff used alone owing to the lower value of absorption capacity for viscose alone. These results confirm the positive effect of the insertion of the optimal quantity of the viscose into the absorbent core. This fact points out the possibility to reduce the quantity of SAP in the core. Thus, the use of viscose fibers in the mixture with fluff and SAP enables to decrease the quantity of the expensive SAP in the core while keeping the equal results of core absorption performance.

For these experiments, the mixture ratio is fixed at $80 / 20$. Fig. (7) illustrates the evolution of absorption capacity and retention values with continuously augmentation of SAP. The blend composed of fluff and acrylic shows a behavior that differs from the mixture of fluff and viscose. As shown, retention values are still the same for different mixture. For absorbent capacity, it continues to rise with the increase of super absorbent polymer. Certainly, the increase of absorption capacity didn't have the same gradient as at ratios under

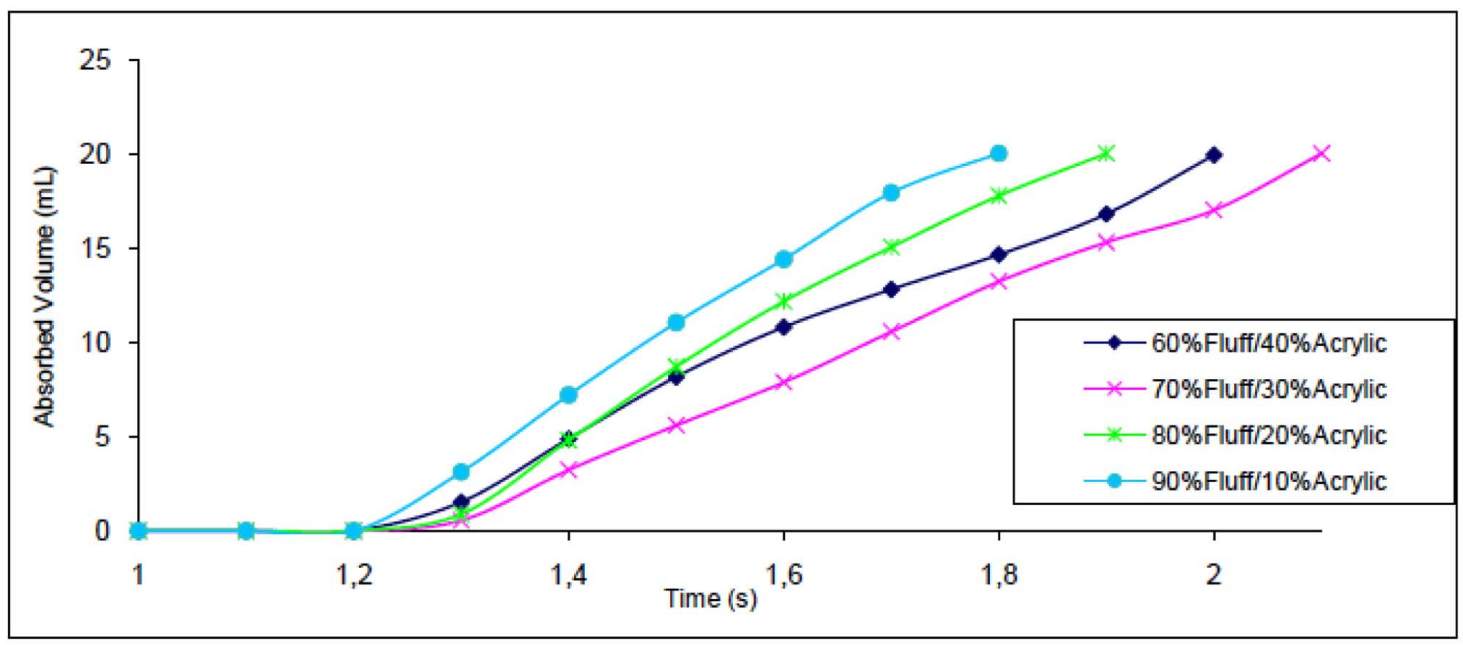

Fig. (5). Influence of acrylic fiber on absorption kinetic of mixture with the fluff. 


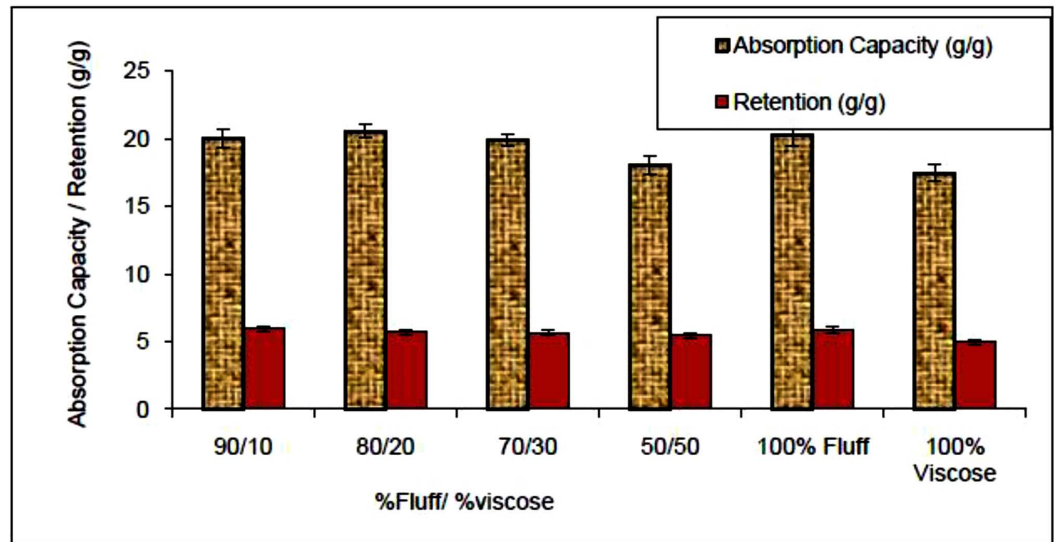

Fig. (6). Retention and absorption capacity for fiber blend with super absorbent ratio.

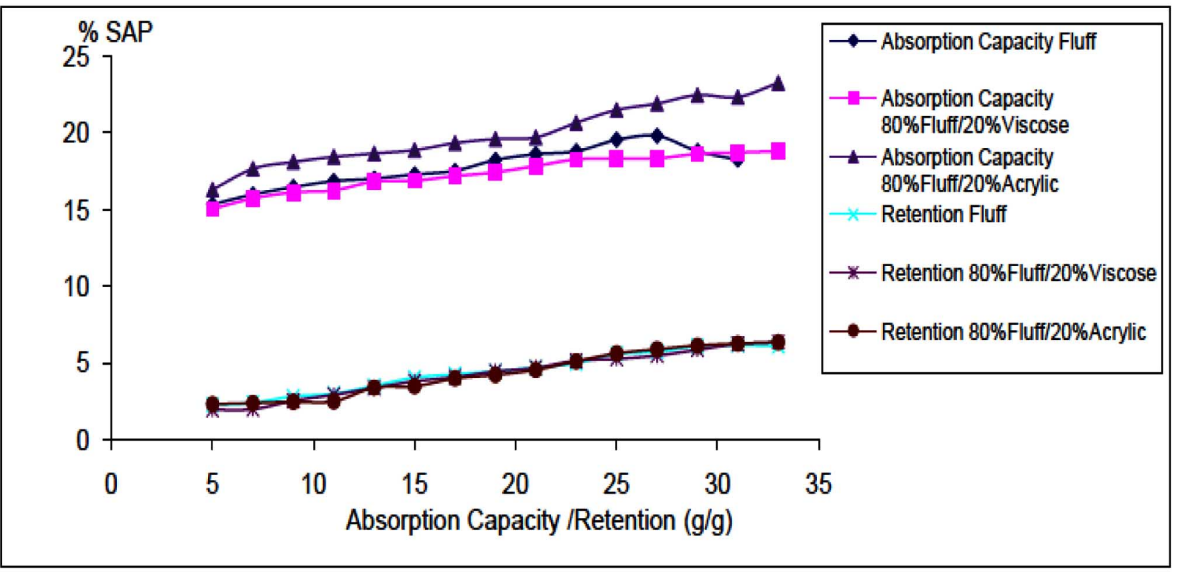

Fig. (7). Retention and absorption capacity for mixture of fluff and viscose/acrylic fiber at different ratio of super absorbent.

$30 \%$ : the increase of absorption capacity is not proportional to the increase of the quantity of SAP in the mixture. It may be supposed that the decrease of the volume of voids and capillaries in the interstitial space and insufficient possibility of the core to absorb, retain and distribute liquid in entire the space of the diaper i.e. there is an effect of "gel blocking" [8] (Fig. 8).

This phenomenon occurs at $25 \%$ of super absorbent content in the case of a blend of fluff and viscose and at $27 \%$

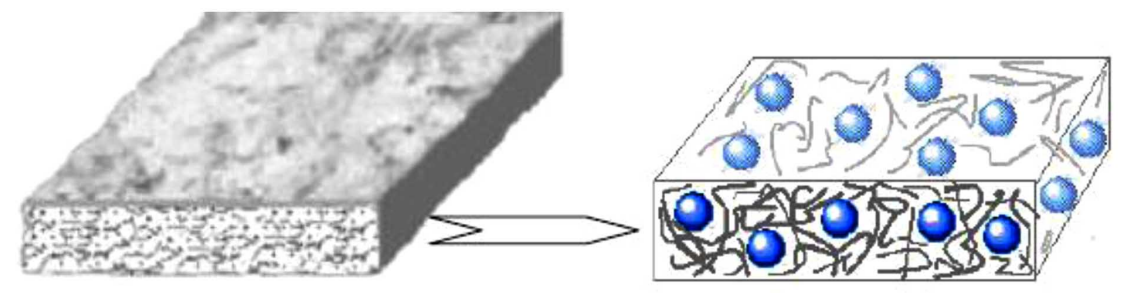

SAP particles repartition in the absorbent core

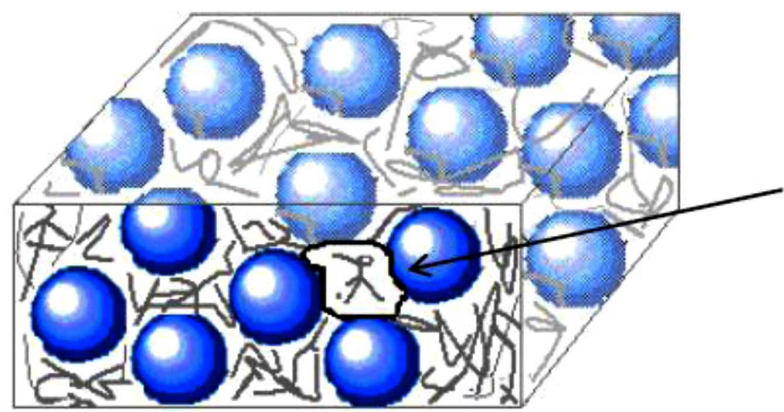

Void volume

decrease after SAP

swelling.

Fig. (8). Schematic representation of gel blocking. 
for the fluff alone. Nevertheless by inserting acrylic, the phenomenon seems to be enabled to occur.

These findings suggest that the fibrous structure composed of cellulosic fiber (fluff) which has a swelling character and a synthetic fiber (acrylic) which is resilient contribute to structure in which changes in the interstitial voids is not too important like other cases. Yet, the swelling of super absorbent takes place but the more porous structure offered by acrylic fibers improves the absorption because it reduces contacts between the swelling particles of SAP. Thus, it may be concluded that the improvement of liquid absorption permits the use of acrylic in the mixture with SAP for production of the core for disposable diapers.

\section{CONCLUSION}

Disposable diapers are products which principal quality is the high absorption and ability to distribute liquid in the whole volume of the absorbing core of the structure. Absorptive properties of the components of the absorbent core determine the behavior of the pad in the process of absorption of liquid.

The main objective of this research was to improve absorption performance of fibrous components by including other fibers, which differ from the fluff by their physicomechanical properties.

The results of the investigation have shown that, the acrylic is able to form a structure that imbibes and retain liquid. The presence of this fiber with the fluff enhances the absorption and retention capacity more than viscose fiber. By the reinforcement of the physical structure, the acrylic promotes the rapidity of absorption by allowing good kinetics of absorption.

With the presence of super absorbent the gel blocking phenomenon caused by the swelling of super absorbent seems to be enabled thanks to the physical structure obtained after adding acrylic to the fibrous component.

\section{ACKNOWLEDGEMENT}

None declared.

\section{CONFLICT OF INTEREST}

None declared.

\section{REFERENCES}

[1] W. Audra, and A. Frank, "Comfort perception of breathable and nonbreathable diapers", Int. Nonw. J., vol. 14, pp. 19-22, Fall 2005.

[2] R. Saminathan, and B. W. Steven, "Modern diaper technology", Tappi J., vol. 79, pp. 173-177, June 1996.

[3] P. Wiertz, "Sustainability report 2007-2008: Absorbent hygiene Products ", 2011, Available from: http://www.edana.org/content/ default.asp?PageID=75\&DocID=2132 [Accessed 10/02/11]

[4] EDANA, "Absorbent hygiene products, training course: Baby diapers, Sanitary Protection and Adult incontinence, vol. 1, 1997. pp. 25-35, 42-51, Available online at [www.edama.org]

[5] L. Darry, "Nonwovens containing immobilized superabsorbent polymer particles", Int. Nonw. J.,vol. 12, pp. 35-39, Fall 2003.

[6] M.G. Kamath, and A., Dahiya, "Absorption properties ", 2004 Available from: http:/www.engr.utk.edu/mse/Textiles/ Absorption.htm [Accessed 13/01/2010].

[7] T.H. Kevin, "Superabsorbent polymers and rewet performance of the internal layers of absorbent disposable products", Tappi J., vol. 74, pp. 205-212, August 1991.

[8] J. Dutkiewicz, "Some Advances in Nonwoven Structures for Absorbancy, Comfort and Aesthetics", AUTEX Res. J., vol. 2, pp. 153-165, September 2002.

[9] L.F. Fryer, and B.S. Gupta, "Determination of pore size distribution in fibrous webs and its impact on absorbency". In: Tappi Proceedings Nonwoven Conference, Charlotte, 1996, pp. 321-327.

[10] C. Rosinskaya, A.Weinberg, and S. Fishman, "Effect of synthetic fibers on the absorption properties of pads", Int. Nonw. J., vol. 11, pp. 11-17, Summer 2002 .

[11] A. Patnaik, and R.S. RengasamyWetting and wicking in fibrous materials. Textile Progress, The Textile Institute, Cambridge England, Vol. 38, No. 1, pp. 1-10, January 2006.

[12] B.S.Gupta, and C.J. Hong, "Changes in web dimensions during fluid uptake and the impact on absorbency", Tappi J., vol. 77, pp. 181-188, December 1994.

Received: June 22, 2011

Revised: September 07, 2011

Accepted: September 10, 2011

(C) Sayeb et al.; Licensee Bentham Open.

This is an open access article licensed under the terms of the Creative Commons Attribution Non-Commercial License (http: //creativecommons.org/licenses/by$\mathrm{nc} / 3.0 /$ ), which permits unrestricted, non-commercial use, distribution and reproduction in any medium, provided the work is properly cited. 